Article

\title{
Hankel Determinants for Univalent Functions Related to the Exponential Function
}

\section{Paweł Zaprawa (D)}

Faculty of Mechanical Engineering, Lublin University of Technology, 20-618 Lublin, Poland; p.zaprawa@pollub.pl

Received: 16 September 2019; Accepted: 25 September 2019; Published: 28 September 2019

check for updates

\begin{abstract}
Recently, two classes of univalent functions $\mathcal{S}_{e}^{*}$ and $\mathcal{K}_{e}$ were introduced and studied. A function $f$ is in $\mathcal{S}_{e}^{*}$ if it is analytic in the unit disk, $f(0)=f^{\prime}(0)-1=0$ and $\frac{z f^{\prime}(z)}{f(z)} \prec e^{z}$. On the other hand, $g \in \mathcal{K}_{e}$ if and only if $z g^{\prime} \in \mathcal{S}_{e}^{*}$. Both classes are symmetric, or invariant, under rotations. In this paper, we solve a few problems connected with the coefficients of functions in these classes. We find, among other things, the estimates of Hankel determinants: $H_{2,1}, H_{2,2}, H_{3,1}$. All these estimates improve the known results. Moreover, almost all new bounds are sharp. The main idea used in the paper is based on expressing the discussed functionals depending on the fixed second coefficient of a function in a given class.
\end{abstract}

Keywords: Hankel determinant; starlike functions; convex functions; exponential function

MSC: 30C45; 30C50

\section{Introduction}

Let $\mathcal{A}$ be the collection of functions of the form

$$
f(z)=z+\sum_{n=2}^{\infty} a_{n} z^{n}
$$

which are analytic in the open unit disk $\Delta=\{z \in \mathbb{C}:|z|<1\}$ and let $\mathcal{S}$ denote the subclass of $\mathcal{A}$ consisting of functions which are univalent in $\Delta$.

Since the early twentieth century many mathematicians have been interested in different problems involving the coefficients of functions $f$ in a given subclass of $\mathcal{A}$. The most important and inspiring problem known as the Bieberbach conjecture was solved by de Branges only 70 years after its formulation. Over the years, many interesting tasks connected with these coefficients appeared. The most important ones were settled by Robertson, Bombieri, Zalcman, Krzyż and Landau. In the 1960s Pommerenke defined the Hankel determinant $H_{k, n}$, for a given $f$ of the form (1), as follows

$$
H_{k, n}=\left|\begin{array}{cccc}
a_{n} & a_{n+1} & \ldots & a_{n+k-1} \\
a_{n+1} & a_{n+2} & \ldots & a_{n+k} \\
\ldots & \ldots & \ldots & \ldots \\
a_{n+k-1} & a_{n+k} & \ldots & a_{n+2 k-2}
\end{array}\right|,
$$

where $n, k \in \mathbb{N}$.

The studies on Hankel determinants are concentrated on estimating $H_{2,2}$ and $H_{3,1}$ for different subclasses of $\mathcal{S}$. These particular determinants can be written as

$$
H_{2,2}=a_{2} a_{4}-a_{3}^{2}
$$


and

$$
H_{3,1}=a_{3}\left(a_{2} a_{4}-a_{3}^{2}\right)+a_{4}\left(a_{2} a_{3}-a_{4}\right)+a_{5}\left(a_{3}-a_{2}^{2}\right) .
$$

Although we know many sharp bounds of $H_{2,2}$ and significantly less sharp bounds of $H_{3,1}$ for some proper subfamilies of $\mathcal{S}$, the sharp results for the whole class $\mathcal{S}$ are not known. Moreover, we are even unable to formulate a reasonable conjecture about it.

Among numerous results for the subclasses of $\mathcal{S}$, we cite only the most important one. In [1], Janteng et al. proved that $\left|H_{2,2}\right| \leq 1$ for $\mathcal{S}^{*}$ and $\left|H_{2,2}\right| \leq 1 / 8$ for $\mathcal{K}$, where $\mathcal{S}^{*}$ and $\mathcal{K}$ are very well known classes of starlike and convex functions. The sharp results for $H_{3,1}$ are difficult to obtain. It is worth citing the sharp bound $\left|H_{3,1}\right| \leq 4 / 135$ for $\mathcal{K}$ and the non-sharp estimate $\left|H_{3,1}\right| \leq 8 / 9$ for $\mathcal{S}^{*}$ obtained by Kowalczyk et al. and Kwon et al., respectively (see, [2,3]).

The definitions of $\mathcal{S}^{*}$ and $\mathcal{K}$ can be written in terms of subordination. Namely

$$
\mathcal{S}^{*}=\left\{f \in \mathcal{S}: \frac{z f^{\prime}(z)}{f(z)} \prec \frac{1+z}{1-z}, z \in \Delta\right\}
$$

and

$$
\mathcal{K}=\left\{f \in \mathcal{S}: 1+\frac{z f^{\prime \prime}(z)}{f^{\prime}(z)} \prec \frac{1+z}{1-z}, z \in \Delta\right\} .
$$

Let us recall that for two functions $f$ and $g$ analytic in $\Delta$, we say that $g$ is subordinated to $f(g \prec f)$, if there exists a function $\omega$ analytic in $\Delta$ with $\omega(0)=0,|\omega(z)|<1$ and such that $g(z)=f(\omega(z))$. The relation $g \prec f$ results in $g(\Delta) \subset f(\Delta)$.

Let $\phi$ be an analytic function such that $\Re \phi(z)>0$ for $z \in \Delta$ with $\phi(0)=1$. Ma and Minda [4] defined the classes of $\mathcal{S}^{*}(\phi)$ and $\mathcal{K}(\phi)$ in the following way

$$
\mathcal{S}^{*}(\phi)=\left\{f \in \mathcal{S}: \frac{z f^{\prime}(z)}{f(z)} \prec \phi(z), z \in \Delta\right\}
$$

and

$$
\mathcal{K}(\phi)=\left\{f \in \mathcal{S}: 1+\frac{z f^{\prime \prime}(z)}{f^{\prime}(z)} \prec \phi(z), z \in \Delta\right\} .
$$

From (5) it is seen that $\mathcal{S}^{*}(\phi)$ reduces to $\mathcal{S}^{*}$ if $\phi(z)=\frac{1+z}{1-z}$. For other specific choices of $\phi$ we obtain for example:

1. if $\phi_{1}(z)=\frac{1+(1-2 \alpha) z}{1-z}$, then $\mathcal{S}^{*}\left(\phi_{1}\right)=\mathcal{S}^{*}(\alpha)$ is the class of starlike functions of order $\alpha$,

2. if $\phi_{2}(z)=\left(\frac{1+z}{1-z}\right)^{\alpha}$, then $\mathcal{S}^{*}\left(\phi_{2}\right)=\mathcal{S}_{\alpha}^{*}$ is the class of strongly starlike functions of order $\alpha$,

3. if $\phi_{3}(z)=\frac{1+A z}{1+B z}$, then $\mathcal{S}^{*}\left(\phi_{3}\right)=\mathcal{S}^{*}[A, B]$ is the class of Janowski starlike functions.

In a similar way we defined the relative subclasses of $\mathcal{K}$.

Recently, Mendiratta et al. [5] discussed $\mathcal{S}_{e}^{*}$ and $\mathcal{K}_{e}$, i.e., the classes $\mathcal{S}^{*}(\phi)$ and $\mathcal{K}(\phi)$ with $\phi(z)=e^{z}$. Various problems, including distortion and growth theorems, radii problems, inclusion relations and coefficient estimates, were discussed there.

In their two following papers Zhang et al. [6] and Shi et al. [7] broadened the range of discussed coefficient problems. They found the coefficient estimates for $a_{n}, n=2,3,4,5$ and the bounds of the following functionals: $a_{2} a_{3}-a_{4}, H_{2,1}, H_{2,2}$ and, as a consequence, $H_{3,1}$. Except for the bounds of $a_{n}$, $n=2,3,4$ and $H_{2,1}$, all results are non-sharp.

In this paper, we improve all non-sharp results mentioned above. The main idea is to express the discussed functionals depending on the second coefficient of $f \in \mathcal{S}_{e}^{*}$ or $g \in \mathcal{K}_{e}$. In fact, the second coefficient of $f \in \mathcal{S}_{e}^{*}$ or $g \in \mathcal{K}_{e}$ can be replaced by the coefficient $p_{1}$ of a corresponding function $P$ with a positive real part. This idea leads to better estimates than those in [6,7]. Moreover, the new bounds of $a_{2} a_{3}-a_{4}$ and $H_{2,2}$ are sharp. 


\section{Auxiliary Lemmas}

To prove our results, we need two lemmas concerning functions in the class $\mathcal{P}$ of functions $P$ such that $\Re P(z)>0$ and $P$ has the Taylor series representation

$$
P(z)=1+\sum_{n=1}^{\infty} p_{n} z^{n}
$$

Lemma 1 ([8]). Let $p_{1} \in[0,2]$. A function $P$ of the form (9) is in $\mathcal{P}$ if and only if

1. $2 p_{2}=p_{1}^{2}+x\left(4-p_{1}^{2}\right)$,

2. $4 p_{3}=p_{1}^{3}+2 p_{1}\left(4-p_{1}^{2}\right) x-p_{1}\left(4-p_{1}^{2}\right) x^{2}+2\left(4-p_{1}^{2}\right)\left(1-|x|^{2}\right) y$,

for some $x$ and $y$ such that $|x| \leq 1,|y| \leq 1$.

Lemma 2 ([4,9]). If $P \in \mathcal{P}$ is of the form (9) and $\mu \in[0,1]$, then the following sharp estimates hold

1. $\left|p_{n+m}-\mu p_{m} p_{n}\right| \leq 2$ for $n, m=1,2, \ldots$,

2. $\left|p_{2}-\mu p_{1}^{2}\right| \leq \begin{cases}2-\mu\left|p_{1}\right|^{2} & , \mu \in[0,1 / 2], \\ 2-(1-\mu)\left|p_{1}\right|^{2} & , \mu \in[1 / 2,1] .\end{cases}$

In fact, the second inequality of Lemma 2 did not appear in [4], but it is a reformulation of the result obtained for bounded functions.

At the end of this section, observe that both classes $\mathcal{S}_{e}^{*}$ and $\mathcal{K}_{e}$ possess a specific type of symmetry. They are invariant (or symmetric) under rotations. Recall that the class $A$ is invariant under rotations when $f$ is in $A$ if and only if $f_{\varphi}(z)=e^{-i \varphi} f\left(z e^{i \varphi}\right), \varphi \in \mathbb{R}$ is also in $A$. A functional $J(f)$ defined for functions $f \in A$ is called invariant under rotations in $A$ if $f_{\varphi} \in A$ and $J(f)=J\left(f_{\varphi}\right)$ for all $\varphi \in \mathbb{R}$. It can be easily checked that $\mathcal{S}_{e}^{*}$ and $\mathcal{K}_{e}$, as well as the functionals $\left|a_{2} a_{3}-a_{4}\right|,\left|H_{2,1}\right|,\left|H_{2,2}\right|$ and $\left|H_{3,1}\right|$ considered in $\mathcal{S}_{e}^{*}$ or in $\mathcal{K}_{e}$, satisfy the above definitions. Due to the symmetry described above, in the considerations we can assume that one coefficient (usually the second one) is a positive real number.

\section{Coefficient Problems for $\mathcal{S}_{e}^{*}$}

It follows from the definition of $\mathcal{S}_{e}^{*}$ that there exists a function $\omega(z)$ with $\omega(0)=0,|\omega(z)|<1$ such that

$$
\frac{z f^{\prime}(z)}{f(z)}=e^{\omega(z)}
$$

Define $P(z)=\frac{1+\omega(z)}{1-\omega(z)}=1+p_{1} z+p_{2} z^{2}+\ldots$. The function $P$ is in $\mathcal{P}$ and (10) is equivalent to

$$
\frac{z f^{\prime}(z)}{f(z)}=\exp \left(\frac{P(z)-1}{P(z)+1}\right)
$$

Now, expanding both sides of (11) in the Taylor series and comparing coefficients at $z^{k}, k=1,2,3,4$, we obtain (see also Formulae (15)-(18) in [7]),

$$
a_{2}=\frac{1}{2} p_{1}, a_{3}=\frac{1}{4}\left(p_{2}+\frac{1}{4} p_{1}^{2}\right), a_{4}=\frac{1}{6}\left(p_{3}+\frac{1}{4} p_{1} p_{2}-\frac{1}{48} p_{1}^{3}\right), a_{5}=\frac{1}{8}\left(p_{4}+\frac{1}{6} p_{1} p_{3}-\frac{1}{12} p_{1}^{2} p_{2}+\frac{1}{144} p_{1}^{4}\right)
$$

Now, we can prove the following theorem.

Theorem 1. If $f \in \mathcal{S}_{e}^{*}$ is given by (1) and $\left|a_{2}\right|=p / 2, p \in[0,2]$, then

1. $\left|a_{3}\right| \leq \frac{1}{4}\left(2+\frac{1}{4} p^{2}\right)$

2. $\left|a_{4}\right| \leq \frac{1}{6}\left(2+\frac{1}{2} p-\frac{1}{48} p^{3}\right)$

3. $\left|a_{5}\right| \leq \frac{1}{8}\left(2+\frac{1}{3} p+\frac{1}{144} p^{4}\right)$. 
Proof. The bound of $a_{3}$ is clear. To obtain the bound of $a_{4}$ and $a_{5}$ it is enough to write

$$
\begin{gathered}
a_{4}=\frac{1}{6}\left[p_{3}+\frac{1}{4} p_{1}\left(p_{2}-\frac{1}{12} p_{1}^{2}\right)\right], \\
a_{5}=\frac{1}{8}\left[p_{4}+\frac{1}{6} p_{1}\left(p_{3}-\frac{1}{2} p_{1} p_{2}\right)+\frac{1}{144} p_{1}^{4}\right]
\end{gathered}
$$

and to apply Lemma 2 .

Corollary 1. If $f \in \mathcal{S}_{e}^{*}$ is given by (1), then

1. $\left|a_{2}\right| \leq 1$

2. $\left|a_{3}\right| \leq \frac{3}{4}$

3. $\left|a_{4}\right| \leq \frac{17}{36}$

4. $\left|a_{5}\right| \leq \frac{25}{72}$.

The first three bounds are sharp.

The function which gives equality in the bounds of $a_{2}, a_{3}$ and $a_{4}$ corresponds to $P(z)=\frac{1+z}{1-z}$, so $\omega(z)=z$. This means that the extremal function is of the form

$$
f_{1}(z)=z \cdot \exp \left(\int_{0}^{z} \frac{e^{\zeta}-1}{\zeta} d \zeta\right), z \in \Delta
$$

so

$$
f_{1}(z)=z+z^{2}+\frac{3}{4} z^{3}+\frac{17}{36} z^{4}+\frac{19}{72} z^{5}+\ldots .
$$

In [5], Mendiratta et al. proved that if $f \in \mathcal{S}_{e}^{*}$, then $\left|a_{3}-a_{2}{ }^{2}\right| \leq \frac{1}{2}$. Although this inequality is sharp, we can easily generalize it by applying Lemma 2 in the following identity $a_{3}-a_{2}^{2}=\frac{1}{4}\left(p_{2}-\frac{3}{4} p_{1}^{2}\right)$.

Theorem 2. If $f \in \mathcal{S}_{e}^{*}$ is given by (1) and $\left|a_{2}\right|=p / 2, p \in[0,2]$, then $\left|a_{3}-a_{2}{ }^{2}\right| \leq \frac{1}{4}\left(2-\frac{1}{4} p^{2}\right)$. The result is sharp.

For sharpness, it is enough to discuss a function $f$ which corresponds to

$$
P_{p}(z)=\frac{1-z^{2}}{1-p z+z^{2}}=1+p z+\left(p^{2}-2\right) z^{2}+\left(p^{3}-3 p\right) z^{3}+\left(p^{4}-4 p^{2}+2\right) z^{4}+\ldots
$$

For this function,

$$
a_{3}-a_{2}^{2}=\frac{1}{4}\left(p_{2}-\frac{3}{4} p_{1}^{2}\right)=\frac{1}{4}\left(\frac{1}{4} p^{2}-2\right) .
$$

Now, we shall improve the estimate $\left|a_{2} a_{3}-a_{4}\right| \leq 0.535 \ldots$ found by Zhang et al. (see, Theorem 2 in [6]).

Theorem 3. If $f \in \mathcal{S}_{e}^{*}$ is given by (1) and $\left|a_{2}\right|=p / 2, p \in[0,2]$, then

$$
\left|a_{2} a_{3}-a_{4}\right| \leq \begin{cases}\frac{1}{288}\left(96-18 p^{2}+13 p^{3}\right) & , p \in[0,4 / 3], \\ \frac{1}{144}\left(48 p-7 p^{3}\right) & , p \in[4 / 3,2] .\end{cases}
$$

Consequently, we get the following corollary.

Corollary 2. If $f \in \mathcal{S}_{e}^{*}$ is given by (1), then

$$
\left|a_{2} a_{3}-a_{4}\right| \leq \frac{8}{9 \sqrt{7}}=0.335 \ldots
$$

The result is sharp. 
Proof of Theorem 3. From (12) and Lemma 1 we obtain

$$
\left|a_{2} a_{3}-a_{4}\right|=\frac{1}{24}\left|4 p_{3}-2 p_{1} p_{2}-\frac{5}{6} p_{1}^{3}\right|=\frac{1}{24}\left|-\frac{5}{6} p_{1}^{3}+\left(4-p_{1}^{2}\right)\left(\left(x-x^{2}\right) p_{1}+2\left(1-|x|^{2}\right) y\right)\right|,
$$

with $x, y$ such that $|x| \leq 1,|y| \leq 1$.

In a view of the invariance of $\left|a_{2} a_{3}-a_{4}\right|$ under rotations, assume that $r=|x| \in[0,1]$ and $p=p_{1} \in[0,2]$. Then,

$$
\left|a_{2} a_{3}-a_{4}\right| \leq \frac{1}{24} h(r),
$$

with

$$
h(r)=\frac{5}{6} p^{3}+2\left(4-p^{2}\right)+p\left(4-p^{2}\right) r-\left(4-p^{2}\right)(2-p) r^{2} .
$$

The function $h(r)$ achieves the greatest value in $[0,1]$ when $r=\frac{p}{2(2-p)}$ if $p \in[0,4 / 3]$ and when $r=1$ if $p \in[4 / 3,2]$.

Proof of Corollary 2. Define $h_{1}(p)=\frac{1}{288}\left(96-18 p^{2}+13 p^{3}\right), p \in[0,4 / 3]$ and $h_{2}(p)=\frac{1}{144}\left(48 p-7 p^{3}\right)$, $p \in[4 / 3,2]$. It is easy to observe that

$$
\max \left\{h_{1}(p), p \in[0,4 / 3]\right\}=\max \left\{h_{1}(0), h_{1}(4 / 3)\right\}=h_{1}(0)=\frac{1}{3}
$$

and

$$
\max \left\{h_{2}(p), p \in[4 / 3,2]\right\}=h_{2}(4 / \sqrt{7})=\frac{8}{9 \sqrt{7}}
$$

which proves (17).

Observe that the equality in (17) holds if $x=-1$ and $p=4 / \sqrt{7}$ in (18). This means that $P \in \mathcal{P}$ is of the form (15) with $p=4 / \sqrt{7}$. In this case,

$$
a_{2} a_{3}-a_{4}=\frac{1}{144}\left(7 p^{3}-48 p\right)=-\frac{8}{9 \sqrt{7}} .
$$

The next theorem improves the bound of $H_{2,2}$ also found by Zhang et al. (see, Theorem 3 in [6]).

Theorem 4. If $f \in \mathcal{S}_{e}^{*}$ is given by (1) and $\left|a_{2}\right|=p / 2, p \in[0,2]$, then

$$
\left|a_{2} a_{4}-a_{3}^{2}\right| \leq \frac{1}{2304}\left(576-48 p^{2}-11 p^{4}\right) .
$$

The bound is sharp.

Consequently, we get the following corollary.

Corollary 3. If $f \in \mathcal{S}_{e}^{*}$ is given by (1), then

$$
\left|a_{2} a_{4}-a_{3}^{2}\right| \leq \frac{1}{4}
$$

The bound is sharp.

Proof of Theorem 4. From (12) we obtain

$$
a_{2} a_{4}-a_{3}^{2}=\frac{1}{2304}\left(192 p_{1} p_{3}-24 p_{1}^{2} p_{2}-13 p_{1}^{4}-144 p_{2}^{2}\right)
$$

By Lemma 1,

$$
a_{2} a_{4}-a_{3}^{2}=\frac{1}{2304}\left[-13 p_{1}^{4}+12 p_{1}^{2}\left(4-p_{1}^{2}\right) x-12\left(12+p_{1}^{2}\right)\left(4-p_{1}^{2}\right) x^{2}+96 p_{1}\left(4-p_{1}^{2}\right)\left(1-|x|^{2}\right) y\right],
$$


where $|x| \leq 1,|y| \leq 1$.

We can assume that $a_{2}=p_{1} / 2$ is a non-negative real number. Applying the triangle inequality and writing $p$ instead of $p_{1}, p \in[0,2]$, we have

$$
\left|a_{2} a_{4}-a_{3}^{2}\right| \leq \frac{1}{2304} h(r),
$$

where

$$
h(r)=13 p^{4}+96 p\left(4-p^{2}\right)+12 p^{2}\left(4-p^{2}\right) r+12\left(4-p^{2}\right)(2-p)(6-p) r^{2}, r=|x| .
$$

However, $h$ is an increasing function of $r \in[0,1]$, so $h(r) \leq h(1)$, which results in (20).

Observe that the equality in (20) holds only when $x=-1$. This means that $P$ is of the form (15). Hence,

$$
a_{2}=\frac{1}{2} p, a_{3}=\frac{1}{16}\left(5 p^{2}-8\right), a_{4}=\frac{1}{288}\left(59 p^{3}-168 p\right),
$$

so

$$
a_{2} a_{4}-a_{3}^{2}=\frac{1}{2304}\left(11 p^{4}+48 p^{2}-576\right) .
$$

For $p=0$ we have $P_{0}(z)=\frac{1-z^{2}}{1+z^{2}}$, so from (11),

$$
\frac{z f^{\prime}(z)}{f(z)}=e^{-z^{2}}
$$

Hence, the corresponding function in $\mathcal{S}_{e}^{*}$ is of the form

$$
f_{0}(z)=z \cdot \exp \left(\int_{0}^{z} \frac{e^{-\zeta^{2}}-1}{\zeta} d \zeta\right)=z-\frac{1}{2} z^{3}+\ldots, z \in \Delta
$$

Finally, we find a new bound of $H_{3,1}$ for the class $\mathcal{S}_{e}^{*}$. In [6] it was proved that $\left|H_{3,1}\right| \leq 0.565 \ldots$ In the succeeding paper Shi et al. showed that $\left|H_{3,1}\right| \leq 0.500 \ldots$ (see, Theorem 1 in [7]). We improve these results essentially in the following way.

Theorem 5. If $f \in \mathcal{S}_{e}^{*}$ is given by (1), then

$$
\left|H_{3,1}\right| \leq 0.385 \ldots
$$

Proof. Applying Theorems 1-4 and the triangle inequality in (4) we obtain

$$
\left|H_{3,1}\right| \leq\left|a_{3}\right| \cdot\left|a_{2} a_{4}-a_{3}{ }^{2}\right|+\left|a_{4}\right| \cdot\left|a_{2} a_{3}-a_{4}\right|+\left|a_{5}\right| \cdot\left|a_{3}-a_{2}{ }^{2}\right| \leq\left\{\begin{array}{l}
k_{1}(p), p \in[0,4 / 3] \\
k_{2}(p), p \in[4 / 3,2]
\end{array}\right.
$$

where

$$
k_{1}(p)=\frac{1}{331776}\left(119808+16128 p-10368 p^{2}+2016 p^{3}+168 p^{4}+72 p^{5}-169 p^{6}\right)
$$

and

$$
k_{2}(p)=\frac{1}{331776}\left(82944+43776 p+5760 p^{2}-6240 p^{3}-2808 p^{4}-61 p^{6}\right) .
$$

The function $k_{2}$ is decreasing for $p \in[4 / 3,2]$. Moreover, $k_{1}$ attains its greatest value in $[0,4 / 3]$, which is equal to $0.385 \ldots$, at $p=1.140 \ldots$. This results in the declared bound. 


\section{Coefficient Problems for $\mathcal{K}_{e}$}

Directly from the definitions of $\mathcal{S}_{e}^{*}$ and $\mathcal{K}_{e}$ it follows that for $f(z)=z g^{\prime}(z)$,

$$
g \in \mathcal{K}_{e} \Leftrightarrow f \in \mathcal{S}_{e}^{*} .
$$

Consequently, if $g \in \mathcal{K}_{e}$,

$$
g(z)=z+b_{2} z^{2}+\ldots
$$

and $f \in \mathcal{S}_{e}^{*}$ is given by (1), then $b_{n}=a_{n} / n$. For this reason, Theorem 1 results in the two following facts.

Theorem 6. If $g \in \mathcal{K}_{e}$ is given by (27) and $\left|b_{2}\right|=p / 4, p \in[0,2]$, then

1. $\left|b_{3}\right| \leq \frac{1}{12}\left(2+\frac{1}{4} p^{2}\right)$

2. $\left|b_{4}\right| \leq \frac{1}{24}\left(2+\frac{1}{2} p-\frac{1}{48} p^{3}\right)$

3. $\left|b_{5}\right| \leq \frac{1}{40}\left(2+\frac{1}{3} p+\frac{1}{144} p^{4}\right)$.

Corollary 4. If $g \in \mathcal{K}_{e}$ is given by (27), then
1. $\left|b_{2}\right| \leq \frac{1}{2}$
2. $\left|b_{3}\right| \leq \frac{1}{4}$
3. $\left|b_{4}\right| \leq \frac{17}{144}$
4. $\left|b_{5}\right| \leq \frac{5}{72}$.

Equalities in the bounds of the first three coefficients hold for $g_{1}$ such that $z g_{1}^{\prime}(z)=f_{1}(z)$, where $f_{1}$ is defined by (13). Hence,

$$
g_{1}(z)=z+\frac{1}{2} z^{2}+\frac{1}{4} z^{3}+\frac{17}{144} z^{4}+\frac{19}{360} z^{5}+\ldots .
$$

Proceeding in the same manner as in Section 3, we obtain results for $b_{3}-b_{2}{ }^{2}, b_{2} b_{3}-b_{4}$ and $b_{2} b_{4}-b_{3}^{2}$.

Theorem 7. If $g \in \mathcal{K}_{e}$ is given by (27) and $\left|b_{2}\right|=p / 4, p \in[0,2]$, then $\left|b_{3}-b_{2}{ }^{2}\right| \leq \frac{1}{12}\left(2-\frac{1}{2} p^{2}\right)$. The result is sharp.

The proof of this theorem is obvious.

Theorem 8. If $g \in \mathcal{K}_{e}$ is given by (27) and $\left|b_{2}\right|=p / 4, p \in[0,2]$, then

$$
\left|b_{2} b_{3}-b_{4}\right| \leq \begin{cases}\frac{1}{4608}\left(384-42 p^{2}+31 p^{3}\right) & , p \in[0,8 / 7], \\ \frac{1}{1152}\left(120 p-29 p^{3}\right) & , p \in[8 / 7,2] .\end{cases}
$$

Consequently, we get the following corollary.

Corollary 5. If $g \in \mathcal{K}_{e}$ is given by (27), then

$$
\left|b_{2} b_{3}-b_{4}\right| \leq \frac{1}{12}
$$

The result is sharp.

Proof of Theorem 8. From (26), (12) and Lemma 1 we obtain

$$
\left|b_{2} b_{3}-b_{4}\right|=\frac{1}{96}\left|4 p_{3}-p_{1} p_{2}-\frac{7}{12} p_{1}^{3}\right|=\frac{1}{96}\left|-\frac{1}{12} p_{1}^{3}+\left(4-p_{1}^{2}\right)\left(\left(\frac{3}{2} x-x^{2}\right) p_{1}+2\left(1-|x|^{2}\right) y\right)\right|,
$$


with $x, y$ such that $|x| \leq 1,|y| \leq 1$.

Assume that $r=|x| \in[0,1]$ and $p=p_{1} \in[0,2]$. Then,

$$
\left|b_{2} b_{3}-b_{4}\right| \leq \frac{1}{96} h(r),
$$

with

$$
h(r)=\frac{1}{12} p^{3}+2\left(4-p^{2}\right)+\frac{3}{2} p\left(4-p^{2}\right) r-\left(4-p^{2}\right)(2-p) r^{2} .
$$

The maximal value of $h(r)$ in $[0,1]$ is achieved if $r=\frac{3 p}{4(2-p)}$ for $p \in[0,8 / 7]$ and if $r=1$ for $p \in[8 / 7,2]$.

Proof of Corollary 5. Define $h_{1}(p)=\frac{1}{4608}\left(384-42 p^{2}+31 p^{3}\right), p \in[0,8 / 7]$ and $h_{2}(p)=\frac{1}{1152}(120 p-$ $\left.29 p^{3}\right), p \in[8 / 7,2]$. It is easy to observe that

$$
\max \left\{h_{1}(p), p \in[0,8 / 7]\right\}=\max \left\{h_{1}(0), h_{1}(8 / 7)\right\}=h_{1}(0)=\frac{1}{12}
$$

and

$$
\max \left\{h_{2}(p), p \in[8 / 7,2]\right\}=h_{2}(\sqrt{40 / 29})=\frac{5 \sqrt{290}}{1044}=0.081 \ldots,
$$

which leads to (32).

The equality in (32) holds if we put $x=0, y=-1$ and $p=0$ into (31). This means that $P(z)=\frac{1-z^{3}}{1+z^{3}}$. Consequently, the extremal function $g_{2}$ is such that $z g_{2}^{\prime}(z)=f_{2}(z)$, where $f_{2}$ is of the form

$$
\frac{z f_{2}^{\prime}(z)}{f_{2}(z)}=e^{-z^{3}}
$$

Hence

$$
f_{2}(z)=z \cdot \exp \left(\int_{0}^{z} \frac{e^{-\zeta^{3}}-1}{\zeta} d \zeta\right)=z-\frac{1}{3} z^{4}+\ldots, z \in \Delta
$$

and

$$
g_{2}(z)=z-\frac{1}{12} z^{4}+\ldots
$$

In the next theorem we improve the bound of $H_{2,2}$ obtained by Shi et al. (see, Theorem 4 in [7]).

Theorem 9. If $g \in \mathcal{K}_{e}$ is given by (27) and $\left|b_{2}\right|=p / 4, p \in[0,2]$, then

$$
\left|b_{2} b_{4}-b_{3}{ }^{2}\right| \leq \frac{1}{4608}\left(128+8 p^{2}-9 p^{4}\right) .
$$

The bound is sharp.

Consequently, we get the following corollary.

Corollary 6. If $g \in \mathcal{K}_{e}$ is given by (27), then

$$
\left|b_{2} b_{4}-b_{3}{ }^{2}\right| \leq \frac{73}{2592}=0.028 \ldots
$$

The bound is sharp.

Proof of Theorem 9. From (12) and (26) we obtain

$$
b_{2} b_{4}-b_{3}{ }^{2}=\frac{1}{4608}\left(48 p_{1} p_{3}-4 p_{1}^{2} p_{2}-3 p_{1}^{4}-32 p_{2}^{2}\right) .
$$


Applying Lemma 1, we get

$$
b_{2} b_{4}-b_{3}^{2}=\frac{1}{4608}\left[-p_{1}^{4}+6 p_{1}^{2}\left(4-p_{1}^{2}\right) x-4\left(8+p_{1}^{2}\right)\left(4-p_{1}^{2}\right) x^{2}+24 p_{1}\left(4-p_{1}^{2}\right)\left(1-|x|^{2}\right) y\right],
$$

where $|x| \leq 1,|y| \leq 1$.

Without loss of generality, we assume that $p_{1}=p \in[0,2]$. Hence,

$$
\left|b_{2} b_{4}-b_{3}^{2}\right| \leq \frac{1}{4608} h(r),
$$

where

$$
h(r)=p^{4}+24 p\left(4-p^{2}\right)+6 p^{2}\left(4-p^{2}\right) r+4\left(4-p^{2}\right)(2-p)(4-p) r^{2}, r=|x| .
$$

Clearly, $h(r) \leq h(1)$, which results in (33).

The equality in (33) holds only when $x=-1$. In this case $P$ is given by (15) and

$$
b_{2}=\frac{1}{4} p, b_{3}=\frac{1}{48}\left(5 p^{2}-8\right), b_{4}=\frac{1}{1152}\left(59 p^{3}-168 p\right),
$$

so

$$
b_{2} b_{4}-b_{3}^{2}=\frac{1}{4608}\left(9 p^{4}-8 p^{2}-128\right) \text {. }
$$

By combining the results presented above we can derive a bound of $H_{3,1}$ when $g \in \mathcal{K}_{e}$.

Theorem 10. If $g \in \mathcal{K}_{e}$ is given by (27), then

$$
\left|H_{3,1}\right| \leq 0.021 \ldots
$$

Proof. From (4) and the triangle inequality it follows that

$$
\left|H_{3,1}\right| \leq\left|a_{3}\right| \cdot\left|a_{2} a_{4}-a_{3}{ }^{2}\right|+\left|a_{4}\right| \cdot\left|a_{2} a_{3}-a_{4}\right|+\left|a_{5}\right| \cdot\left|a_{3}-a_{2}{ }^{2}\right| \leq\left\{\begin{array}{l}
k_{1}(p), p \in[0,8 / 7] \\
k_{2}(p), p \in[8 / 7,2],
\end{array}\right.
$$

where

$$
k_{1}(p)=\frac{1}{26542080}\left(528384+82944 p-52416 p^{2}-1296 p^{3}-3192 p^{4}+210 p^{5}-1427 p^{6}\right)
$$

and

$$
k_{2}(p)=\frac{1}{6635520}\left(86016+66816 p+6336 p^{2}-16224 p^{3}-5808 p^{4}-173 p^{6}\right) .
$$

The function $k_{2}$ is decreasing for $p \in[8 / 7,2]$. Moreover, $k_{1}$ attains its greatest value in $[0,8 / 7]$, which is equal to $0.021 \ldots$, at $p=0.715 \ldots$. This results in the declared bound.

\section{Concluding Remark}

The main aim of this paper was to improve the already known bounds of the Hankel determinants for $f \in \mathcal{S}_{e}^{*}$ and $g \in \mathcal{K}_{e}$. For this reason, we did not focus on finding the best possible bounds of $a_{5}$ and $b_{5}$. The results presented in Theorem 1 and in Theorem 6 were sufficient for our purpose. It is worth adding that both estimates can be improved if a more precise inequality than $\left|p_{3}-\frac{1}{2} p_{1} p_{2}\right| \leq 2$ for $P \in \mathcal{P}$ is applied. In [10] it was proved that

$$
\left|p_{3}-\frac{1}{2} p_{1} p_{2}\right| \leq \begin{cases}\frac{1}{16} p^{3}-\frac{3}{8} p^{2}+2, & p \in\left[0, \frac{4}{3}\right] \\ 2 p-\frac{1}{2} p^{3}, & p \in\left[\frac{4}{3}, 2\right] .\end{cases}
$$


Although this consequently gives

$$
\left|a_{5}\right| \leq \frac{13}{44} \quad \text { for } \quad f \in \mathcal{S}_{e}^{*}
$$

and

$$
\left|b_{5}\right| \leq \frac{13}{220} \text { for } g \in \mathcal{K}_{e},
$$

the final bounds of $H_{3,1}$ for $\mathcal{S}_{e}^{*}$ and $\mathcal{K}_{e}$ are only slightly better than these from Theorem 5 and Theorem 10.

Funding: The project/research was financed in the framework of the project Lublin University of Technology - Regional Excellence Initiative, funded by the Polish Ministry of Science and Higher Education (contract no.030/RID/2018/19)

Conflicts of Interest: The author declares no conflict of interest. The funders had no role in the design of the study; in the collection, analyses, or interpretation of data; in the writing of the manuscript, or in the decision to publish the results.

\section{References}

1. Janteng, A.; Halim, S.A.; Darus, M. Hankel determinant for starlike and convex functions. Int. J. Math. Anal. 2007, 13, 619-625.

2. Kowalczyk, B.; Lecko, A.; Sim, Y.J. The sharp bound for the Hankel determinant of the third kind for convex functions. Bull. Aust. Math. Soc. 2018, 97, 435-445. [CrossRef]

3. Kwon, O.S.; Lecko, A.; Sim, Y.J. The bound of the Hankel determinant of the third kind for starlike functions. Bull. Malays. Math. Sci. Soc. 2019, 42, 767-780. [CrossRef]

4. Ma, W.; Minda, D. A unified treatment of some special classes of univalent functions. In Proceedings of the Conference on Complex Analysis, Tianjin, China, 19-23 June 1992; pp. 157-169.

5. Mendiratta, R.; Nagpal, S.; Ravichandran, V. On a subclass of strongly starlike functions associated with exponential function. Bull. Malays. Math. Sci. Soc. 2015, 38, 365-386. [CrossRef]

6. Zhang, H.-Y.; Tang, H.; Niu, X.-M. Third-order Hankel determinant for certain class of analytic functions related with exponential function. Symmetry 2018, 10, 501. [CrossRef]

7. Shi, L.; Srivastava, H.M.; Arif, M.; Hussain, S.; Khan, H. An investigation of the third Hankel determinant problem for certain subfamilies of univalent functions involving the exponential function. Symmetry 2019, 11, 598. [CrossRef]

8. Libera, R.J.; Złotkiewicz, E.J. Early coefficients of the inverse of a regular convex function. Proc. Am. Math. Soc. 1982, 85, 225-230. [CrossRef]

9. Hayami, T.; Owa, S. Generalized Hankel determinant for certain classes. Int. J. Math. Anal. 2010, 4, 2573-2585.

10. Trąbka-Więctaw, K.; Zaprawa, P. On the coefficient problem for close-to-convex functions. Turk. J. Math. 2018, 42, 2809-2818. [CrossRef] 